\title{
Viabilidade da obtenção de polpa de acerola (malpighia spp) microencapsulada e liofilizada: Uma revisão
}

\author{
Viability of obtaining microencapsulated and lyophilized acerola pulp (malpighia spp): A review \\ Viabilidad de obtener pulpa de acerola (malpighia spp) microencapsulada y liofilizada: Una \\ revisión
}

Recebido: 01/02/2021 | Revisado: 05/02/2021 | Aceito: 09/02/2021 | Publicado: 16/02/2021

\author{
Bruno Henrique Figueiredo Saqueti \\ ORCID: https://orcid.org/0000-0002-1118-4605 \\ Universidade Estadual de Maringá, Brasil \\ E-mail: bruno_saqueti@outlook.com \\ Eloize Silva Alves \\ ORCID: https://orcid.org/0000-0002-3340-8374 \\ Universidade Estadual de Maringá, Brasil \\ E-mail: eloizeetaus@gmail.com \\ Isadora Boaventura Sá Ponhozi \\ ORCID: https://orcid.org/0000-0001-7230-161X \\ Universidade Estadual de Maringá, Brasil \\ E-mail: isa.ponhozi@gmail.com \\ Pâmela Alves Castilho \\ ORCID: https://orcid.org/0000-0003-1601-4864 \\ Universidade Estadual de Maringá, Brasil \\ E-mail: pamela.alvescastilho@gmail.com \\ Matheus Campos Castro \\ ORCID: https://orcid.org/0000-0002-9918-1491 \\ Universidade Estadual de Maringá, Brasil \\ E-mail: 1996mcastro@gmail.com \\ Patrícia Magalhães Souza \\ ORCID: https://orcid.org/0000-0001-5916-0744 \\ Universidade Estadual de Maringá, Brasil \\ E-mail: patrícia.magalhaes11@hotmail.com \\ Paula Montanhini Favetta \\ ORCID: https://orcid.org/0000-0001-7373-6412 \\ Universidade Paranaense, Brasil \\ E-mail: nutripaulafavetta@gmail.com \\ Jesuí Vergílio Visentainer \\ ORCID: https://orcid.org/0000-0003-3412-897X \\ Universidade Estadual de Maringá, Brasil \\ E-mail: jesuivv@gmail.com \\ Oscar de Oliveira Santos \\ ORCID: https://orcid.org/0000-0002-9631-8480 \\ Universidade Estadual de Maringá, Brasil \\ E-mail: oliveirasantos.oscardeoliveira@gmail.com
}

\begin{abstract}
Resumo
O objetivo desta revisão é fornecer uma compreensão profunda das técnicas que induzem os processamentos de qualidade da acerola e preservação de seus compostos bioativos. Foi possível concluir que a acerola é um fruto com grande potencial antioxidante, devido seus compostos bioativos, sendo necessário a aplicação de tecnologias como a microencapsulação e liofilização para conservação dos mesmos. Novos estudos se tornam necessários para a avaliação do comportamento da polpa de acerola em diferentes parâmetros de aplicação, além da realização de analises físicoquímica, "in vitro" e "in vivo".
\end{abstract}

Palavras-chave: Tecnologia; Agente encapsulante; Secagem; Ácido Ascórbico.

\begin{abstract}
The purpose of this review is to provide a deep understanding of the techniques that induce the quality processing of acerola and the preservation of its bioactive compounds. It was possible to conclude that acerola is a fruit with great antioxidant potential, due to its bioactive compounds, requiring the application of technologies such as microencapsulation and lyophilization to preserve them. Further studies are needed to assess the behavior of acerola pulp in different application parameters, in addition to performing physical-chemical analyzes, in vitro and in vivo.
\end{abstract}


Keywords: Technology; Encapsulating agent; Drying; Ascorbic acid.

\section{Resumen}

El propósito de esta revisión es proporcionar una comprensión profunda de las técnicas que inducen el procesamiento de calidad de la acerola y la preservación de sus compuestos bioactivos. Se pudo concluir que la acerola es una fruta con gran potencial antioxidante, debido a sus compuestos bioactivos, requiriendo la aplicación de tecnologías como la microencapsulación y la liofilización para conservarlos. Se necesitan más estudios para evaluar el comportamiento de la pulpa de acerola en diferentes parámetros de aplicación, además de realizar análisis físico-químicos, "in vitro" e "in vivo".

Palabras clave: Tecnología; Agente encapsulante; El secado; Ácido ascórbico.

\section{Introdução}

Acerola (Malpighia spp) é uma planta de origem da América Central. Esta fruta é conhecida como uma excelente fonte alimentar de vitamina C, também contém compostos fitoquímicos, como carotenoides e compostos fenólicos (Mezadri et al., 2008). Compostos fenólicos presentes na acerola, possuem propriedades bioquímicas e farmacológicas, como antioxidante (Hossen et al., 2017), atividades antivirais, anticancerígenas e anti-inflamatórias (Silva, Duarte, \& Barrozo, 2019). O ácido ascórbico (vitamina C) é presente em elevadas concentrações, é uma das vitaminas mais importantes na prevenção do escorbuto, e é associado à regulação do colesterol (Ashor et al., 2018; Singh et al., 2018).

O beneficiamento da acerola consiste na obtenção de produtos como polpas, sucos, concentrados, doces, compostas e geleias, com tecnologia adequada para cada processamento (Bortolotti et al., 2013). O principal produto obtido são as polpas congeladas, processamento importante na atividade agroindustrial e que valorizam os frutos, evitando desperdícios e diminuindo as perdas pela comercialização do fruto na sua forma "in natura" (Do Nascimento, 2018).

Técnicas vem sendo empregadas para conservação de compostos bioativos em polpas de frutas, como a microencapsulação, que tem como função proteger os compostos de interesse do ambiente externo, utilizando agentes encapsulantes apropriados como revestimento (Paulo, \& Santos, 2017). A técnica de microencapsulação pode ser classificada em três grupos: técnicas físicas, técnicas químicas e técnicas físico-químicas, cada método apresenta vantagens e desvantagens em relação a matriz que se deseja encapsular (Ozkan et al., 2019).

$\mathrm{Na}$ técnica de microencapsulação associam-se processos de secagem como a liofilização, esta técnica promove a remoção de água de misturas congeladas por sublimação em baixas temperaturas e sob vácuo (Ezhilarasi, Karthik, Chhanwal, \& Anandharamakrishnan, 2013). Garante qualidade superior a outras técnicas, mantendo a biofuncionalidade e uma vida útil mais longa aos compostos bioativos, não afetando as propriedades sensoriais (Tarone, Cazarin, \& Junior, 2020).

A estabilidade dos compostos bioativos da polpa de acerola demonstra ser afetada pelo $\mathrm{pH}$, exposição a luz, oxigênio e temperatura (Rezende, Nogueira, \& Narain, 2017). O principal fitoquímico é o ácido ascórbico, vitamina hidrossolúvel e sensível a altas temperaturas que se caracteriza como um antioxidante instável, facilmente oxidável, cujas ligações podem ser quebradas pelo oxigênio, substâncias alcalinas e temperaturas elevadas (Manela-azulay et al., 2003). A microencapsulação de substâncias quimicamente sensíveis, como o ácido ascórbico, por spray drying tem se mostrado viável para obtenção de sucos desidratados, concentrando as substâncias ativas típicas e protegendo-as em uma matriz polimérica contra oxidação (Ferrari, Ribeiro, \& Aguirre, 2012). O objetivo desta revisão é fornecer uma compreensão profunda das técnicas que induzem os processamentos de qualidade da acerola e preservação de seus compostos bioativos por microencapsulação e liofilização.

\section{Metodologia}

Foram realizadas buscas nas bases de dados Google Scholar, Scielo e Science direct com palavras chaves para busca de dados: Acerola, Malpighia, microencapsulation, freeze-drying, lyophilization, pulp of fruits. Assim, sendo uma busca metodológica qualitativa em uma revisão bibliográfica (Pereira, Shitsuka, Pereira, \& Shitsuka, 2018). 


\section{Resultados e Discussão}

\section{Coleta de dados}

Realizando uma busca nas bases de dados já citadas, e utilizando as palavras chaves: Acerola, microencapsulation e lyophilization foram encontrados 469, 5328, 10099 trabalhos publicados, respectivamente, os dados encontrados estão dispostos na Figura 1 abaixo.

Figura 1. Gráfico de publicações ao longo dos anos, utilizando as palavras chave: Acerola, Lyophilization e Microencapsulation.

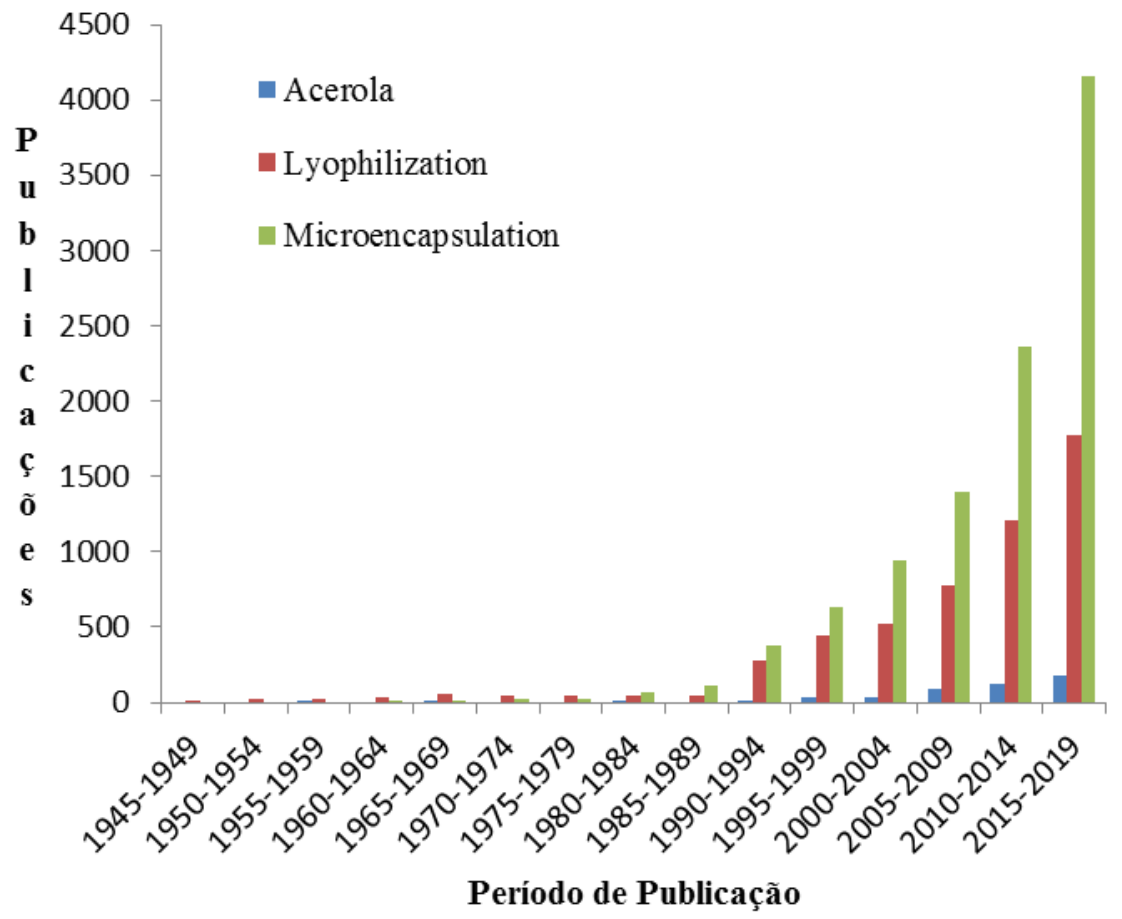

Fonte: Autores (2021).

Analisando a Figura 1 é possivel observar que é crescente o interesse por pesquisas, nas quais tratam da acerola, do processo de liofilização ou de microencapsulação, a partir do ano de 2010 nota-se um interesse maior pronunciado por pesquisas por esses termos, sendo que o termo que mais teve pesquisas conduzidas foi o de microencapsulação, isso se deve ao fato, dos cientistas estarem preocupados, em entender melhor o processo de microencapsulação, assim como utiliza-lo na conservação de compostos bioativos, logo realizar uma revisão bibliografica levando em conta a viabilidade desses processos (microencapsulaçao e liofilização) é de extrema importância, para o universo acâdemico se posicionar frente as novas descobertas.

\section{Acerola (Malpighia spp)}

A Acerola (Malpighia spp) é pertencente a família Malpighiaceae contendo cerca de 45 espécies, entre pequenas árvores e arbustos (Mezadri et al., 2008). Nativa da América central, e cultivada principalmente no Brasil, México, índia e alguns países do sudeste da Ásia (Quoc, Hoa, Ngoc, \& Phi, 2015). O Brasil é o maior produtor, consumidor e exportador de Acerola, destacando a região nordeste por cerca de $70 \%$ da produção nacional do fruto, devido às condições climáticas e composição do solo favorável para cultura (Mamede et al., 2009). 
$\mathrm{O}$ fruto quando maduro é uma drupa, possui um fino epicarpo, um mesocarpo (polpa) que quantifica em média de 75\% do peso do fruto total e um endocarpo trilobado envolvendo uma semente com 3 a $5 \mathrm{~mm}$ de diâmetro, uma forma oval e dois cotilédones (Moura et al., 2018). Os frutos de Acerola são bem conhecidos por sua elevada concentração de ácido ascórbico (Vitamina C), compostos fitoquímicos como compostos fenólicos, incluindo derivados do ácido benzóico, fenilpropanóides, flavonóides, antocianinas e carotenoides. (Belwal et al., 2018). Vitaminas como tiamina, riboflavina, niacina e carotenoides (Pró-vitamina A) e minerais como cálcio, fósforo ferro, magnésio e potássio (Moura et al., 2018).

O ácido ascórbico (vitamina C) é presente em elevadas concentrações na Acerola, sendo uma das vitaminas mais importantes na prevenção do escorbuto, e associada à regulação do colesterol (Ashor et al., 2019). O importante papel da vitamina $\mathrm{C}$ como antioxidante, onde ela promove a neutralização dos radicais livres, que tem a função de provocar o processo de envelhecimento precoce, além dos riscos de desenvolvimento de doenças crônicas (Goel, \& Agarwal, 2020). A Vitamina C é caracterizada por sua degradabilidade no processamento e preparação de alimentos podendo ser afetada pelo $\mathrm{pH}$, exposição a luz, oxigênio e temperatura (Herbig, \& Renard, 2017). Na Acerola a concentração de Vitamina C pode variar entre os cultivares, região, fatores ambientais e com as fases de maturação, sendo alto em frutos verdes $(1,90 \mathrm{~g} / 100 \mathrm{~g}$ de suco) e diminuem durante o amadurecimento (0,97 g / $100 \mathrm{~g}$ de suco em frutas maduras) (Belwal et al., 2018).

Sua composição centesimal é formada pelo teor de umidade $90,5 \%$, lipídios $0,2 \%$, proteína $0,9 \%$, carboidrato $8,0 \%$, fibra alimentar 1,5\%, cinzas 0,4\% (Lima, 2006). Em sua fração lipídica, foram identificados os seguintes ácidos graxos: oleico (31,9\%), linoleico $(29,2 \%)$, palmítico $(21,8 \%)$, esteárico $(13,9 \%)$ e linolênico $(1,3 \%)$. Os principais açúcares são a frutose e a glicose, em menor concentração a sacarose (Moura et al., 2018). Seu sabor agradável e altos níveis de compostos antioxidantes tornam-se atrativos ao consumo de diversas formas, como, frutas frescas ou produtos industrializados (polpas, sucos, geleias, concentrados, sorvete, xaropes e licores) (Silva; Duarte, \& Barrozo, 2016).

A capacidade antioxidante de polpas e sucos de acerola foram avaliados por Mezadri et al. (2008), os valores obtidos para o suco de acerola foram superiores aos relatados para outros sucos de frutas particularmente ricos em polifenóis, como sucos de morango, uva e maçã. Cinco compostos polifenólicos diferentes foram identificados nas amostras por meio de HPLC e detecção de arranjo de diodos: ácido clorogênico, galato de epigalocatequina, epicatequina, procianidina B1 e rutina, sendo os dois últimos predominantes.

Efeitos terapêuticos do suco de acerola, como um possível agente antioxidante, em animais expostos a uma dieta hipocalórica foi investigado, esses efeitos protetores podem estar relacionados a uma complexa mistura de antioxidantes presentes na acerola que possui altos níveis de ácido ascórbico, carotenoides e polifenóis (incluindo quercetina e rutina) (Schreckinger, Lotton, Lila, \& de Mejia, 2010). Leffa et al. (2015) sugerem suplementação alimentar com suco de acerola maduro ou industrial e a suplementação de suco de acerola verde, devido a efeitos benéficos observados auxiliando na redução do estresse oxidativo e poder diminuir a genotoxicidade em condições obesogênicas.

O efeito sinérgico antioxidante e anti-inflamatório foi avaliado no estudo de Souza et al. (2020), onde a mistura formula em pó de Malpighia emarginata DC (Acerola) e Camellia sinensis L. foi capaz de modular os parâmetros redox nas células durante a resposta inflamatória in vitro, além disso, o co-tratamento com a mistura foi capaz de modular a resposta inflamatória alterando a secreção de citocinas. Os resultados justificam o uso adicional das misturas para enriquecimento de produtos para prevenção de doenças crônicas. É demonstrado em pesquisas diversas atividades biológicas como: atividade antioxidante, antimutagênica, antidiabética, antitumoral e hepatoprotetora (Paz et al., 2015; Düsman, Almeida, Tonin, \& Vicentini, 2016; Barbalho et al., 2011; Luveena, Karthiayini, \& Sreekumar, 2012). 


\section{Processamento da Acerola}

Pelo fato da acerola ser uma fonte natural rica em ácido ascórbico e outros compostos, ferramentas e técnicas são estudadas de forma a agregar valor à fruta por meio de produtos. Geleia, suco concentrado, compota, suplementos, são algumas das formas de comercializar a fruta processada. $\mathrm{O}$ estudo dos pigmentos presentes na acerola como corante alimentício natural também pode apresentar resultados promissores para aplicações agroindustriais e farmacêuticas (Prakash, \& Baskara, 2018). Além disso, outras aplicações como a transformação da acerola em pó, empregada em cosméticos ou seu uso em forma de cápsulas de vitamina C podem trazer benefícios (Le, Luong, Le, \& Cabaltica, 2018). Considerando que a fruta é perecível e possui vida de prateleira curta, estes processamentos podem ser aplicados de forma a estender seu prazo de validade e possibilitar o aproveitamento de seus nutrientes de diferentes formas (Prakash, \& Baskara, 2018; Le, Luong, Le, \& Cabaltica, 2018).

Os componentes presentes na acerola possuem propriedades antioxidantes e benéficas para a saúde, entretanto, tanto o uso de temperaturas nos processamentos quanto o armazenamento prolongado podem fazer com que haja a perda de nutrientes. Estudos são realizados de forma a avaliar o efeito dos tratamentos nos compostos bioativos dos alimentos, com a finalidade de reduzir as perdas das propriedades nutricionais. As condições de armazenamento influenciam nas taxas de perdas dos componentes, sendo que a redução de compostos bioativos se dá de forma mais intensa durante o armazenamento à temperatura ambiente ou sob refrigeração do que à menores temperaturas como $-18 \pm 2{ }^{\circ} \mathrm{C}$ (Le, Luong, Le, \& Cabaltica, 2018).

A pasteurização térmica é conhecida por sua contribuição na segurança e por prolongar a vida de prateleira de alimentos por meio da desnaturação das enzimas e inativação dos microrganismos. Entretanto, sabe-se que pode afetar a qualidade nutricional dos alimentos. Desta forma, ao submeter suco de acerola a um processamento ultrassônico, foi observado que o suco apresentou resultados benéficos em relação à estabilidade e propriedade nutricionais (Santos, Rodrigues, \& Fernandes, 2018).

Wurlitzeret al. (2019) em um estudo sobre os efeitos da pasteurização e armazenamento sob refrigeração nas características químicas e sensorial de formulações de sucos com acerola, concluíram que a pasteurização à $85^{\circ} \mathrm{C}$ por $30 \mathrm{~s}$ seguido de envase a quente, não apresentou efeitos prejudiciais aos componentes, qualidade sensorial e aceitabilidade de misturas de sucos com acerola, embora o tempo de armazenamento tenha interferido em parâmetros como teor de ácido ascórbico, polifenóis e carotenoides.

Silva, Crispim, \& Vieira (2017), ao estudarem parâmetros de degradação cinética e termodinâmica para antocianinas em polpa de acerola aquecida a diferentes temperaturas, encontraram resultados que indicam que a energia de ativação da degradação apresentou-se muito dependente da temperatura, sendo que os resultados indicaram um processo de degradação relativamente lento.

Ribeiro et al. (2019), ao estudarem os efeitos das variáveis no processo de secagem por pulverização em suco de acerola e seriguela, concluíram que o uso da secagem por pulverização é um método eficiente e capaz de fornecer compostos benéficos à saúde, e o uso da acerola como um potencial agente enriquecedor em sucos em pó ao propiciar um alto teor de ácido ascórbico. Assim como, Rezende, Nogueira, \& Narain (2018), ao encapsularem os extratos de compostos bioativos de resíduo de acerola por pulverização e liofilização, concluíram que o tratamento por spray apresentou melhores resultados em relação à concentrações de alguns compostos bioativos e atividade antioxidante.

\section{Microencapsulação}

A microencapsulação é definida como um processo pelas quais pequenas partículas ou gotículas são cercadas por um revestimento em uma matriz homogênea ou heterogênea, produzindo pequenas cápsulas com muitas propriedades úteis (Kuck, \& Noreña, 2016). Microcápsulas são geralmente partículas de tamanho variando de 1 a 1000 microns (Ye, Georges, \& 
Selomulya, 2018). O material a ser encapsulado pode ser chamado de fase interna, material do núcleo, preenchimento ou agente ativo, enquanto o material encapsulante pode ser referido como membrana, material transportador, revestimento, material de parede ou fase externa (Dhakal \& He, 2020).

Para o processo de microencapsulação ser eficiente, devem-se considerar diversos fatores como: peso molecular e carga elétrica (estrutura molecular), ebulição e ponto de fusão (estado físico), atividade microbiana (estrutura biológica), dissolubilidade e área de contato, estabilidade química e propriedades ópticas (Ye, Georges, \& Selomulya, 2018). A fase interna ou agente encapsulado pode variar de um núcleo liquido com constituintes dispersos ou dissolvidos e um núcleo sólido que pode ser uma mistura de constituintes diferentes, podendo ser os próprios compostos bioativos, estabilizadores, diluentes e compostos que tem propriedade de acelerar, retardar a taxa de liberação (Aguiar, Estevinho, \& Santos, 2016).

Os materiais de revestimento ideais devem ser biocompatíveis, biodegradáveis, atóxicos e de baixos custos (Matté, \& Rosa, 2013). Além disso, deve ter boa qualidade emulsificante, baixa viscosidade em alta concentração e boas características de solubilidade e formação de rede, outra característica para escolha de agentes encapsulantes é a capacidade de direcionar com precisão os locais alvos do trato gastrointestinal para liberação dos compostos bioativos, devem ser resistentes a acidez e condições enzimáticas do estômago tendo capacidade de adesão apenas na região esperada (Shishir et al., 2018).

Um dos agentes encapsulantes mais utilizados são os polissacarídeos, polímeros naturais de carboidratos, formados por monossacarídeos com ligações glicosídicas e que facilmente são sintetizados por plantas, algas e alguns microorganismos (Fathi, Martin, \& Mcclements, 2014). È um material de baixo custo, alta solubilidade em água e estrutura estável, os principais utilizados são a maltodextrina, goma arábica, quitosana, $\beta$ ciclodextrina e alginato (Ye, Georges, \& Selomulya, 2018).

A maltodextrina é um composto a partir de polímeros vegetais hidrolisados, obtida da fécula da mandioca, por hidrólise parcial enzimática e beneficiada através de processo tecnológico adequado, e apresenta-se na forma de pó sendo totalmente solúvel em água. É utilizado como excipiente farmacêutico, agente aglutinante, aglomerante, retentor da umidade, umectante, dispersante e ligante (Batistuzzo, Itaya, \& Eto, 2006). A maltodextrina é classificada como um carboidrato de alto índice glicêmico, em estudos sob sua aplicação, é considerado um carboidrato complexo, pois faz com que a glicose passe para a circulação sanguínea de forma mais lenta, devido seu peso molecular (Sapata, Fayh, \& Oliveira, 2016). Diferentes métodos podem ser usados na microencapsulação são avaliados a partir da aplicação, os métodos podem ser dívidas em três categorias: Métodos físicos, químicos e físico-químicos. Diferentes métodos de secagem são utilizados (Rebello, 2009).

\section{Conservação de alimentos por Secagem}

A secagem é um processo indispensável na indústria alimentícia, visto que a retirada da água do produto ocasiona numa mudança na concentração dos compostos, promovendo estabilidade microbiana, e consequentemente obtendo vantagens como aumento da vida de prateleira do alimento, diminuição do custo de embalagem, menor peso e vantagens ambientais. A segurança e a nutrição são parâmetros que os consumidores não conseguem avaliar visualmente, entretanto, devem ser assegurados pelo produtor, atendendo suas expectativas (Lewicki, 2006).

O processo de secagem ocorre, em grande parte das vezes, em duas etapas. Primeiramente, por meio de uma taxa constante, a umidade interna passa para a superfície do produto na mesma taxa que ocorre a evaporação. Posterior a isto, a taxa de secagem sofre uma queda quando o produto chega a um ponto crítico de umidade. Este ponto crítico depende das características de cada produto (Cohen \& Yang, 1995).

Fazendo o uso da desidratação, a qualidade do produto final pode ser avaliada por diferentes aspectos, como propriedades físicas, químicas e sensoriais. A escolha do processo e parâmetros utilizados na secagem podem ser os responsáveis pelas alterações ocorridas no produto em relação a sua qualidade e quantidade de seus compostos. Assim, é necessário avaliar cuidadosamente antes da escolha de qual método será utilizado (Michalska et al., 2016). As variáveis devem 
ser controladas durante o processamento para que seja preparado de forma a resultar em um produto com a qualidade esperada pelo consumidor. Métodos pós secagem podem ser aplicados para que haja a manutenção da qualidade durante o armazenamento (Lewicki, 2006).

A desidratação por meios térmicos é usada desde a antiguidade e um dos métodos mais utilizados para preservação de alimentos (Lewicki, 2006; Mujumdar \& Jangam, 2011). A utilização de altas temperaturas na desidratação pode ocasionar na diminuição da qualidade do produto. Visto que os consumidores estão cada vez mais exigentes, esperando produtos de alta qualidade, sem nenhuma perda das características sensoriais e com facilidade de manuseio, novas técnicas estão sendo desenvolvidas de forma a utilizar menores temperaturas e menores tempos de exposição, melhorando os resultados obtidos em relação às características finais do produto (Cohen \& Yang, 1995).

Os métodos de secagem iniciaram-se por meio da energia solar. Com o avanço tecnológico, outras formas foram desenvolvidas, como a secagem em bandeja, em estufa, em tambor, extrusão, spray dryer, liofilização, micro-ondas, entre outras (Vega-Mercado, Góngora-Nieto, \& Barbosa-Cánovas, 2001).

A liofilização é muito usada para obter produtos com vida de prateleira viável. (Nireesha et al., 2013). Ela ocorre principalmente em duas etapas, sendo elas o congelamento do produto, seguido da remoção do gelo por sublimação direta sob pressão reduzida (Vega-Mercado, Góngora-Nieto, \& Barbosa-Cánovas, 2001).

O processo de liofilização, por conta da utilização primeiramente do congelamento, não acarreta em grandes danificações resultantes do uso do calor à estrutura. Além disso, também proporciona que não ocorra muito prejuízo na qualidade sensorial do produto, visto que a remoção dos cristais de gelo por sublimação permite que a estrutura seja reidratada rapidamente (Cohen \& Yang, 1995). Por meio da liofilização, o produto no estado sólido sofre poucas alterações, por conta das reações físico-químicas serem desaceleradas ou até inibidas, aumentando a estabilidade e facilitando o manuseio no transporte e armazenamento do produto (Nireesha et al., 2013).

Por fim, é importante ressaltar que, para selecionar o melhor método de secagem para o produto, é preciso conhecer suas características e composição (Vega-Mercado, Góngora-Nieto, \& Barbosa-Cánovas, 2001). Além disso, os pontos positivos e negativos de cada processo de secagem devem ser analisados, para que o processo seja projetado de forma a melhorar a eficácia e obter a qualidade esperada para o produto (Lewicki, 2006).

\section{Conclusão}

Foi possível concluir que a Acerola é um fruto com grande potencial antioxidante, devido seus compostos bioativos. A degradação de seus compostos pode ser evitada por métodos emergentes como a microencapsulação associada com a secagem por liofilização, onde a baixa temperatura e o vácuo evita a perda dos mesmos. Novos estudos se tornam necessários para a avaliação do comportamento da polpa de acerola em diferentes parâmetros de aplicação, além da realização de analises físico-química, "in vitro" e "in vivo".

\section{Agradecimentos}

Os autores agradecem ao apoio ao grupo de pesquisa APLE-A, Coordenação de Aperfeiçoamento de Pessoal Superior (CAPES) e Conselho Nacional de Desenvolvimento Científico e Tecnológico (CNPq) pela assistência financeira.

\section{Referências}

Aguiar, J., Estevinho, B. N., \& Santos, L. (2016). Microencapsulation of natural antioxidants for food application-The specific case of coffee antioxidants-A review. Trends in food science \& technology, 58, 21-39. 
Ashor, A. W., Brown, R., Keenan, P. D., Willis, N. D., Siervo, M., \& Mathers, J. C. (2019). Limited evidence for a beneficial effect of vitamin C supplementation on biomarkers of cardiovascular diseases: an umbrella review of systematic reviews and meta-analyses. Nutrition Research, 61, 1-12.

Barbalho, S. M., Damasceno, D. C., Spada, A. P. M., Palhares, M., Martuchi, K. A., Oshiiwa, M., \& Silva, V. S. D. (2011). Evaluation of glycemic and lipid profile of offspring of diabetic Wistar rats treated with Malpighia emarginata juice. Experimental diabetes research, 2011.

Batistuzzo, J., Itaya, M., \& Eto, Y. (2006). Formulário Médico Farmacêutico, $3^{a}$ edição, editora Pharmabooks. São Paulo, 585.

Belwal, T., Devkota, H. P., Hassan, H. A., Ahluwalia, S., Ramadan, M. F., Mocan, A., \& Atanasov, A. G. (2018). Phytopharmacology of Acerola (Malpighia spp.) and its potential as functional food. Trends in food science \& technology, 74, 99-106.

Bortolotti, C. T., Santos, K. G., Francisquetti, M. C., Duarte, C. R., \& Barrozo, M. A. (2013). Hydrodynamic study of a mixture of West Indian cherry residue and soybean grains in a spouted bed. The Canadian Journal of Chemical Engineering, 91(11), 1871-1880.

Cohen, J. S., \& Yang, T. C. (1995). Progress in food dehydration. Trends in Food Science \& Technology, 6(1), 20-25.

do Nascimento, J. F., dos Santos Barroso, B., Tostes, E. D. S. L., da Silva, A. D. S. S., \& da Silva Júnior, A. C. S. (2018). Análise físico-química de polpas de acerola (Malpighia glabra L.) artesanais e industriais congeladas. PubVet, 12, 131.

Dhakal, S. P., \& He, J. (2020). Microencapsulation of vitamins in food applications to prevent losses in processing and storage: a review. Food Research International, 109326.

Düsman, E., Almeida, I. V., Tonin, L. T. D., \& Vicentini, V. E. P. (2016). In vivo antimutagenic effects of the Barbados cherry fruit (Malpighia glabra Linnaeus) in a chromosomal aberration assay. Genet Mol Res, 15(4).

Ezhilarasi, P. N., Karthik, P., Chhanwal, N., \& Anandharamakrishnan, C. (2013). Nanoencapsulation techniques for food bioactive components: a review. Food and Bioprocess Technology, 6(3), 628-647.

Fathi, M., Martin, A., \& McClements, D. J. (2014). Nanoencapsulation of food ingredients using carbohydrate based delivery systems. Trends in food science \& technology, 39(1), 18-39.

Ferrari, C. C., Ribeiro, C. P., \& Aguirre, J. M. D. (2012). Spray drying of blackberry pulp using maltodextrin as carrier agent. Brazilian journal of food technology, 15(2), 157-165.

Goel, P., \& Agarwal, D. (2020). A Strong Antioxidant: Ascorbic acid or Vitamin C is an active ingredient of Indian Gooseberry (Emblica officinalis). Structure, 4(5), 6.

Herbig, A. L., \& Renard, C. M. (2017). Factors that impact the stability of vitamin C at intermediate temperatures in a food matrix. Food Chemistry, 220, 444451.

Hossen, M. S., Ali, M. Y., Jahurul, M. H. A., Abdel-Daim, M. M., Gan, S. H., \& Khalil, M. I. (2017). Beneficial roles of honey polyphenols against some human degenerative diseases: a review. Pharmacological Reports, 69(6), 1194-1205.

Kuck, L. S., \& Noreña, C. P. Z. (2016). Microencapsulation of grape (Vitis labrusca var. Bordo) skin phenolic extract using gum Arabic, polydextrose, and partially hydrolyzed guar gum as encapsulating agents. Food chemistry, 194, 569-576.

Le, T. T., Luong, Q. H., Le, T. Q., \& Cabaltica, A. D. (2018). Changes of total polyphenolics and vitamin C in acerola during storage and spray drying process. Acta horticulturae.

Leffa, D. D., da Silva, J., Daumann, F., Dajori, A. L. F., Longaretti, L. M., Damiani, A. P., ... \& de Andrade, V. M. (2014). Corrective effects of acerola (Malpighia emarginata DC.) juice intake on biochemical and genotoxical parameters in mice fed on a high-fat diet. Mutation Research/Fundamental and Molecular Mechanisms of Mutagenesis, 770, 144-152.

Lewicki, P. P. (2006). Design of hot air drying for better foods. Trends in Food Science \& Technology, 17(4), 153-163.

Lima, D. M. (2006). Tabela brasileira de composição de alimentos-TACO: versão 2. NEPA/UNICAMP.

Luveena, A. A., Karthiayini, K., \& Sreekumar, K. P. (2012). Effect of Malphigia glabra (Acerola berry) in acetaminophen induced hepatic injury in rats. International Journal of Research in Phytochemistry and Pharmacology, 2(1), 45-51.

Mamede, M. E. D. O., Miranda, M. D. P. S., Ritzinger, R., Godoy, R. C. B. D., \& Velozo, E. D. S. (2009). Physico-chemical and sensorial evaluation of new varieties of acerola. British Food Journal.

Manela-Azulay, M., Mandarim-de-Lacerda, C. A., Perez, M. D. A., Filgueira, A. L., \& Cuzzi, T. (2003). Vitamina C. Anais brasileiros de dermatologia, 78(3), 265-272.

Matté, G. M., \& Rosa, S. (2013). A tecnologia da microencapsulação através das microesferas de quitosana. Revista Iberoamericana de polímeros, 14(5), 206218.

Mezadri, T., Villaño, D., Fernández-Pachón, M. S., García-Parrilla, M. C., \& Troncoso, A. M. (2008). Antioxidant compounds and antioxidant activity in acerola (Malpighia emarginata DC.) fruits and derivatives. Journal of Food Composition and analysis, 21(4), 282-290.

Michalska, A., Wojdyło, A., Lech, K., Łysiak, G. P., \& Figiel, A. (2016). Physicochemical properties of whole fruit plum powders obtained using different drying technologies. Food chemistry, 207, 223-232.

Moura, C. F., Oliveira, L. D. S., de Souza, K. O., da Franca, L. G., Ribeiro, L. B., de Souza, P. A., \& de Miranda, M. R. (2018). Acerola-Malpighia emarginata. In Exotic fruits (pp. 7-14). Academic Press. 
Mujumdar, A. S., \& Jangam, S. V. (2011). Some innovative drying technologies for dehydration of foods. Proceedings of ICEF, Athens, Greece, 555-556.

Nireesha, G. R., Divya, L., Sowmya, C., Venkateshan, N. N. B. M., \& Lavakumar, V. (2013). Lyophilization/freeze drying-an review. International journal of novel trends in pharmaceutical sciences, 3(4), 87-98.

Ozkan, G., Franco, P., De Marco, I., Xiao, J., \& Capanoglu, E. (2019). A review of microencapsulation methods for food antioxidants: Principles, advantages, drawbacks and applications. Food Chemistry, 272, 494-506.

Paz, M., Gúllon, P., Barroso, M. F., Carvalho, A. P., Domingues, V. F., Gomes, A. M., \& Delerue-Matos, C. (2015). Brazilian fruit pulps as functional foods and additives: Evaluation of bioactive compounds. Food Chemistry, 172, 462-468.

Pereira, A. S., Shitsuka, D. M., Pereira, F. J., \& Shitsuka, R. (2018). Metodologia da pesquisa científica. [eBook]. Santa Maria. Ed. UAB / NTE / UFSM. Recuperado de https://repositorio.ufsm.br/bitstream/handle/1/15824/Lic_Computacao_Metodologia-Pesquisa-Cientifica.pdf?sequence=1.

Prakash, A., \& Baskaran, R. (2018). Acerola, an untapped functional superfruit: a review on latest frontiers. Journal of food science and technology, 55(9), 3373-3384.

Quoc, L. P. T., Hoa, D. P., Ngoc, H. T. B., \& Phi, T. T. Y. (2015). Effect of Xanthan gum Solution on the Preservation of Acerola (Malpighia glabra L.). Cercetari Agronomice in Moldova, 48(3), 89-97.

Rebello, F. D. F. P. (2009). Microencapsulação de ingredientes alimentícios. Revista Agrogeoambiental, 1(3).

Rezende, Y. R. R. S., Nogueira, J. P., \& Narain, N. (2017). Comparison and optimization of conventional and ultrasound assisted extraction for bioactive compounds and antioxidant activity from agro-industrial acerola (Malpighia emarginata DC) residue. LWT-Food Science and Technology, 85, $158-169$.

Rezende, Y. R. R. S., Nogueira, J. P., \& Narain, N. (2018). Microencapsulation of extracts of bioactive compounds obtained from acerola (Malpighia emarginata DC) pulp and residue by spray and freeze drying: Chemical, morphological and chemometric characterization. Food Chemistry, 254, 281-291.

Ribeiro, C. M. C. M., Magliano, L. C. D. S. A., Costa, M. M. A. D., Bezerra, T. K. A., Silva, F. L. H. D., \& Maciel, M. I. S. (2019). Optimization of the spray drying process conditions for acerola and seriguela juice mix. Food Science and Technology, 39, 48-55.

Santos, V. O., Rodrigues, S., \& Fernandes, F. A. (2018). Improvements on the stability and vitamin content of acerola juice obtained by ultrasonic processing. Foods, 7(5), 68 .

Sapata, K. B., Fayh, A. P. T., \& Oliveira, A. R. D. (2006). Efectos del consumo previo de carbohidratos sobre la respuesta de glicemia y desempeño. Revista Brasileira de medicina do esporte, 12(4), 189-194.

Schreckinger, M. E., Lotton, J., Lila, M. A., \& de Mejia, E. G. (2010). Berries from South America: a comprehensive review on chemistry, health potential, and commercialization. Journal of medicinal food, 13(2), 233-246.

Shishir, M. R. I., Xie, L., Sun, C., Zheng, X., \& Chen, W. (2018). Advances in micro and nano-encapsulation of bioactive compounds using biopolymer and lipid-based transporters. Trends in Food Science \& Technology, 78, 34-60.

Silva, P. B., Duarte, C. R., \& Barrozo, M. A. S. (2019). A novel system for drying of agro-industrial acerola (Malpighia emarginata DC) waste for use as bioactive compound source. Innovative Food Science \& Emerging Technologies, 52, 350-357.

Silva, N. L., Crispim, J. M., \& Vieira, R. P. (2017). Kinetic and thermodynamic analysis of anthocyanin thermal degradation in acerola (Malpighia emarginata DC) pulp. Journal of Food Processing and Preservation, 41(4), e13053.

Souza, N. C., de Oliveira Nascimento, E. N., de Oliveira, I. B., Oliveira, H. M. L., Santos, E. G. P., Mata, M. E. R. M. C., \& de Bittencourt Pasquali, M. A. (2020). Anti-inflammatory and antixidant properties of blend formulated with compounds of Malpighia emarginata DC (acerola) and Camellia sinensis L.(green tea) in lipopolysaccharide-stimulated RAW 264.7 macrophages. Biomedicine \& Pharmacotherapy, $128,110277$.

Tarone, A. G., Cazarin, C. B. B., \& Junior, M. R. M. (2020). Anthocyanins: New techniques and challenges in microencapsulation. Food Research International, 133, 109092.

Vega-Mercado, H., Góngora-Nieto, M. M., \& Barbosa-Cánovas, G. V. (2001). Advances in dehydration of foods. Journal of food engineering, 49(4), 271-289.

Wurlitzer, N. J., Dionísio, A. P., Lima, J. R., dos Santos Garruti, D., da Silva Araújo, I. M., da Rocha, R. F. J., \& Maia, J. L. (2019). Tropical fruit juice: Effect of thermal treatment and storage time on sensory and functional properties. Journal of food science and technology, 56(12), 5184-5193.

Ye, Q., Georges, N., \& Selomulya, C. (2018). Microencapsulation of active ingredients in functional foods: From research stage to commercial food products. Trends in Food Science \& Technology, 78, 167-179. 